\title{
OPERATOR MODELS FOR HILBERT LOCALLY $C^{*}$-MODULES
}

\author{
AURELian GHEONDEA
}

Abstract. We single out the concept of concrete Hilbert module over a locally $C^{*}$-algebra by means of locally bounded operators on certain strictly inductive limits of Hilbert spaces. Using this concept, we construct an operator model for all Hilbert locally $C^{*}$-modules and, as an application, we obtain a direct construction of the exterior tensor product of Hilbert locally $C^{*}$-modules. These are obtained as consequences of a general dilation theorem for positive semidefinite kernels invariant under an action of a $*$-semigroup with values locally bounded operators. As a by-product, we obtain two Stinespring type theorems for completely positive maps on locally $C^{*}$-algebras and with values locally bounded operators.

Mathematics subject classification (2010): Primary 47A20, Secondary 46L89, 46E22, 43A35.

Keywords and phrases: Locally Hilbert space, inductive limit, projective limit, locally $C^{*}$-algebra, Hilbert locally $C^{*}$-module, positive semidefinite kernel, $*$-semigroup, invariant kernel, completely positive map, reproducing kernel.

\section{REFERENCES}

[1] G. Allan, On a class of locally convex algebras, Proc. London Math. Soc. 15 (1965), 399-421.

[2] C. Apostol, $b^{*}$-Algebras and their representations, J. London Math. Soc. 33 (1971), 30-38.

[3] W. B. ARVESON, The harmonic analysis of automorphism groups, in Operator Algebras and Automorphisms, Proc. Symp. Pure Math., vol. 38:1, pp. 199-269, Amer. Math. Soc. 1982.

[4] W. B. ARVESon, Dilation theory yesterday and today, in A Glimpse at Hilbert Space Operators, pp. 99-123, Oper. Theory Adv. Appl., vol. 207, Birkhäuser Verlag, Basel 2010.

[5] S. AY, A. GHEONDEA, Representations of *-semigroups associated to invariant kernels with values continuously adjointable operators, Integr. Equ. Oper. Theory 87 (2017), 263-307.

[6] D. E. Evans, J. T. Lewis, Dilations of Irreducible Evolutions in Algebraic Quantum Theory, Dublin Institute for Advanced Studies, Dublin, 1977.

[7] D. GaşPaR, P. GaŞPAR, N. LuPA, Dilations on locally Hilbert spaces, in Topics in Mathematics, Computer Science and Philosophy, pp. 107-122, Presa Universitară Clujeană, Cluj-Napoca 2008.

[8] A. GHEONDEA, Dilations of some VH-spaces operator valued kernels, Integr. Equ. Oper. Theory, 74 (2012), 451-479.

[9] A. Grothendieck, Topological Vector Spaces, Gordon and Breach, Montreux 1973.

[10] A. YA. Helems KiI, Banach and Locally Convex Algebras, Oxford University Press, New York, 1993.

[11] A. Inoue, Locally $C^{*}$-algebras, Mem. Fac. Sci. Kyushu Univ. Ser. A, 25 (1971), 197-235.

[12] M. JoiţA, Locally von Neumann algebras, Bull. Math. Soc. Sci. Math. Roumanie (N.S.) 42 (90) (1999), 51-64.

[13] M. JoIŢA, Strict completely positive maps between locally $C^{*}$-algebras and representations on Hilbert modules, J. London Mat. Soc., 2 (66) (2002), 421-432.

[14] M. JoIŢA, Tensor products of Hilbert modules over locally $C^{*}$-algebras, Czech. Math. J. 54 (2004), 727-737.

[15] M. JoIŢA, Hilbert Modules over Locally $C^{*}$-Algebras, Editura Universităţii Bucureşti, Bucureşti 2006.

[16] D. J. Karia, Y. M. PARmar, Operators on locally Hilbert space, J. Analysis, 23 (2015), 59-73.

[17] G. G. Kasparov, Hilbert $C^{*}$-modules: theorems of Stinespring and Voiculescu, J. Operator Theory, 4 (1980), 133-150.

[18] Y. Komura, Some examples on locally convex spaces, Math. Annalen, 153 (1964), 150-162. 
[19] G. KöTHE, Topological Vector Spaces, I, Second edition, Springer-Verlag, Berlin 1983.

[20] E. C. LANCE, Hilbert $C^{*}$-Modules. A toolkit for operator algebraists, London Mathematical Society Lecture Note Series, vol. 210, Cambridge University Press, Cambridge 1995.

[21] A. Mallios, Hermitian K-theory over topological *-algebras, J. Math. Anal. Appl. 106 (1985), 454-539.

[22] G. J. Murphy, $C^{*}$-Algebras and Operator Theory, Academic Press, Boston 1990.

[23] G. J. Murphy, Positive definite kernels and Hilbert $C^{*}$-modules, Proc. Edinburgh Math. Soc. 40 (1997), 367-374.

[24] K. R. Parthasaraty, K. Schmidt, Positive-Definite Kernels, Continous Tensor Products and Central Limit Theorems of Probability Theory, Lecture Notes in Mathematics, vol. 272, Berlin: SpringerVerlag, 1972.

[25] N. C. PhiLliPs, Inverse limits of $C^{*}$-algebras, J. Operator Theory, 19 (1988), 159-195.

[26] K. SCHMÜDGEN, Über LMC*-Algebren, Math. Nachr. 68 (1975), 167-182.

[27] Z. Sebestyen, Every $C^{*}$-seminorm is automatically submultiplicative, Period. Math. Hun., 10 (1979), 1-8.

[28] W. F. Stinespring, Positive functions on $C^{*}$-algebras, Proc. Amer. Math. Soc. 6 (1955), 211-216.

[29] F. H. SZAFranieC, Murphy's positive definite kernels and Hilbert $C^{*}$-modules reorganized, in Banach Center Publications, vol. 89, pp. 275-295, Warszawa 2010.

[30] D. VoICUlesCu, Dual algebraic structures on operator algebras related to free products, J. Operator Theory, 17 (1987), 85-98.

[31] Yu. I. Zhuraev, F. Sharipov, Hilbert modules over locally $C^{*}$-algebras, arXiv: 0011053v3 [math.OA].

[32] B. Sz.-NAGY, Prolongement des transformations de l'espace de Hilbert qui sortent de cet espace, in Appendice au livre "Leçons d'analyse fonctionnelle" par F. Riesz et B. Sz.-Nagy, pp. 439-573, Akademiai Kiado, Budapest, 1955. 